3494.IAC-06-A1.P.1.05

\title{
THE EFFECTS OF A NOVEL "FLUID-LOADING" STRATEGY COMBINING CREATINE AND GLYCEROL ON FLUID RETENTION AND DISTRIBUTION IN HUMANS
}

\author{
Chris Easton ${ }^{1}$ \\ ${ }^{1}$ Space Research Laboratory, University of Glasgow, United Kingdom \\ c.easton.1@research.gla.ac.uk \\ Alyson Calder ${ }^{1,2}$ \\ ${ }^{2}$ Department of Anaesthetics, Glasgow Royal Infirmary, United Kingdom \\ alysoncalder@doctors.org.uk \\ David B. Kingsmore ${ }^{1,3}$ \\ ${ }^{3}$ Department of Surgery, Gartnavel General Hospital, United Kingdom \\ davidkingsmore@hotmail.com \\ Yannis P. Pitsiladis ${ }^{1}$ \\ Y.Pitsiladis@bio.gla.ac.uk
}

\begin{abstract}
The effects of three "fluid-loading" strategies combining creatine (Cr) and glycerol (Gly) on fluid retention and distribution were examined. Three 7-day $\mathrm{Cr}$ and Gly loading protocols (LP) were examined: LP1 $-10 \mathrm{~g} \mathrm{Cr}$ and $75 \mathrm{~g}$ of glucose in 1 litre (L) of water (x 2 daily) and a bolus of $1 \mathrm{~g}$ Gly $\mathrm{kg}^{-1} 2.5$ hours prior to testing $(\mathrm{n}=6)$; LP2 $-10 \mathrm{~g} \mathrm{Cr}, 1 \mathrm{~g}$ Gly $\mathrm{kg}^{-1}$ and $75 \mathrm{~g}$ of glucose in $1 \mathrm{~L}$ of water (x 2 daily) and final supplement consumed 3 hours prior to testing $(\mathrm{n}=6)$; LP3 - as LP2 but final supplement consumed 5 hours prior to testing $(\mathrm{n}=23)$. Only body mass increased (mean \pm s.d.) by $1.59 \pm 0.21 \mathrm{~kg}(\mathrm{P}<0.01)$ post-LP1 and by $1.20 \pm 0.37 \mathrm{~kg}(\mathrm{P}<0.01)$ post-LP2. Post-LP3, body mass increased by $0.97 \pm 0.28 \mathrm{~kg}(\mathrm{P}<0.01)$ with significant increases in total body water $(0.87 \pm 0.21 \mathrm{~L} ; \mathrm{P}$ $<0.01$ ), intra-cellular and extra-cellular water and plasma volume. Combining $\mathrm{Cr}$ and Gly is potentially a clinically useful "fluid-loading" strategy which merits further evaluation for application in spaceflight.
\end{abstract}

\section{INTRODUCTION}

Space travel leads to severe physiological stresses that have profound effects on the wellbeing of astronauts [1]. Many studies have attempted to quantify the exact nature of the problems encountered by astronauts during and on return from prolonged space travel, though few practical solutions have been offered. Astronauts routinely experience a reduction in body mass of 1-3 $\mathrm{kg}$ on return from space flight $[2,3]$ with losses up to $10 \mathrm{~kg}$ reported after long-duration missions [4]. The cause of this loss in body mass is believed to be multifactorial, with insufficient fluid and energy intake and losses in musculoskeletal mass considered the main contributors [1]. Several studies have reported a significant reduction in total body water (TBW) on return from space travel [4-6]. This water loss may largely be accounted for by loss of body mass [4] but also reflects water loss unrelated to muscle atrophy. Microgravity has been shown to remove all hydrostatic gradients within the body [7], resulting 
in a cephalad fluid shift of approximately 1-2 L and a further shift of fluid from extra-cellular to intracellular water compartments [2]. In addition, there is indirect evidence to suggest that the thirst response is reduced in space to such an extent that fluid lost through respiration and sweating is not adequately replaced, resulting in a reduction in TBW and plasma volume by up to $17 \%[2,3,8]$. The extent of plasma volume reduction incurred during space travel may be further exacerbated by the practice of "voluntary dehydration" undertaken by some astronauts prior to both take-off and re-entry to avoid the need to urinate [9]. The effects of a significant reduction in plasma volume may be profound, for example decreasing the thermoregulatory capacity of the body, resulting in an increased heart rate and core temperature during exercise and a reduced exercise tolerance [10]. Additionally, up to two thirds of astronauts suffer from orthostatic intolerance (inability to maintain a standing posture) on return to earth [11] due to the combined effects of reduced central venous pressure [12] and skeletal muscle atrophy that ensues from a lack of static exercise associated with prolonged exposure to microgravity [13].

One experimental approach to maintaining plasma volume during space travel has been to infuse isotonic saline. In a terrestrial environment this maintained central blood volume, resulting in an increased skin blood flow and associated convective heat loss [14]. The medical expertise required to insert and maintain a venous infusion and the restricted mobility that would result, makes this method of rehydration impractical during space travel. Currently, dehydration prior to re-entry into the earth's atmosphere is treated by oral intake of sodium chloride and water [15]. However, this treatment has limited efficacy and may be detrimental in astronauts who have dehydrationinduced hypernatremia. While these current fluid strategies may be partially successful in replacing some of the fluid loss after dehydration has occurred, it may be more desirable to "fluid-load" prior to take-off to minimise subsequent dehydration during flight. Pre-hydrating prior to space flight by ingesting water or electrolyte solutions is ineffective at acutely expanding plasma volume as "excess" fluid ingested is rapidly filtered and excreted by the kidneys [16]. Two hydrating agents have shown promise in promoting water retention, glycerol (1,2,3-propanetriol) (Gly) and creatine (Cr). Supplementation with Gly or $\mathrm{Cr}$ has consistently produced a modest fluid retention of 400 to $800 \mathrm{ml}$ [e.g. 17,18]. Seifert et al. [19] reported a $710 \mathrm{ml}$ increase in mean TBW after Gly ingestion, including a $385 \mathrm{ml}$ increase in interstitial fluid and $225 \mathrm{ml}$ increase in intra-cellular fluid, a plasma expansion of $100 \mathrm{ml}$. In addition, Gly has been shown to effectively minimise an exercise-induced reduction in plasma volume [20] and a reduction in thermal and cardiovascular strain during heat stress [17,21,22]. Ingestion of $\mathrm{Cr}$ has also been shown to have substantial hydrating effects [18], although the exact mechanisms of this remain uncertain. However, unlike the whole body hydrating effects of Gly, $\mathrm{Cr}$ retains fluid predominantly in the intra-cellular fluid compartments [18]. Like Gly, oral $\mathrm{Cr}$ supplementation attenuates the rise in heart rate and core temperature during exercise, effects that have been 
attributed to an increase in intra-cellular water (ICW) resulting in an increased specific heat capacity of the body $[18,23]$.

It seems plausible that a Gly-induced increase in extra-cellular water (ECW) coupled with a $\mathrm{Cr}$-mediated increase in ICW could have a synergistic effect resulting in a much larger increase in TBW than if either supplement was consumed alone. However, the potential implications for fluid balance of simultaneously ingesting two fluid retaining agents are unknown. Therefore, this present series of studies were carried out to examine the effects of three "fluid-loading" strategies that combined $\mathrm{Cr}$ and Gly on fluid retention and distribution. The overall aim was to develop an effective "fluid-loading" strategy for use during conditions of restricted water access, such as during space travel.

\section{MATERIALS AND METHODS}

\subsection{Subjects}

35 endurance-trained males (Table 1) were invited to take part in the present studies that were approved by the local Ethics Committee and performed according to the code of ethics of the World Medical Association (Declaration of Helsinki). Subjects were interviewed to ensure that they had not taken $\mathrm{Cr}$ for at least 8 weeks prior to each study. The investigators did not reveal prior to the interview that subjects would be excluded if they had supplemented with $\mathrm{Cr}$ in the previous 8 weeks. The subjects were fully informed of any risks and discomforts associated with the experiments before giving their written informed consent to participate.

\subsection{Experimental design}

Each study design consisted of two 7 day supplementation periods with measurements obtained pre- and postsupplementation. Subjects followed their normal diet during each supplementation period. Subjects were asked to minimise caffeine intake to lessen any possible confounding effect(s) of caffeine on $\mathrm{Cr}$ supplementation [24]. Each subject was required to follow one of three loading protocols (i.e. LP1, LP2, LP3).

\subsection{Loading protocols}

\subsubsection{Loading protocol 1 (LP1)}

Subjects $(n=6)$ underwent a preliminary baseline test before receiving either combined $\mathrm{Cr}$ and Gly or placebo for 7 days followed by a post-supplementation test. Subsequent to this, subjects who received $\mathrm{Cr} / \mathrm{Gly}$ in the first regimen received placebo and vice versa during a second 7 day supplementation regimen followed by a repeat test (i.e. a total of 3 tests). Cr/Gly supplementation consisted of $11.4 \mathrm{~g}$ of $\mathrm{Cr}^{\mathrm{H}} \mathrm{H}_{2} \mathrm{O}$ (equivalent to $10 \mathrm{~g}$ $\mathrm{Cr}$ ) and $70 \mathrm{~g}$ of glucose polymer made up in $1 \mathrm{~L}$ of warm water and consumed twice daily for 6 days and a bolus of $1 \mathrm{~g}$ Gly $\mathrm{kg}^{-1}$ body mass diluted in $500 \mathrm{ml}$ of water with $125 \mathrm{ml}$ unsweetened diluting juice 2 hours prior to the start of the test. This $\mathrm{Cr}$ supplementation protocol has been shown to increase resting muscle phosphocreatine levels within 5 days [25]. Each supplement was made immediately prior to consumption in order to prevent degradation of $\mathrm{Cr}$ to creatinine. The placebo consisted of $85 \mathrm{~g}$ of glucose polymer made up in $1 \mathrm{~L}$ of warm water and consumed twice daily for 6 days and a bolus of $500 \mathrm{ml}$ of water with $125 \mathrm{ml}$ unsweetened diluting juice 2 hours prior to testing. All supplements 


\begin{tabular}{lcc}
\hline \multicolumn{1}{c}{ LP1 } & $(\mathrm{n}=6)$ & \\
& & \\
Age (yr) & $29 \pm 5$ & \\
Height $(\mathrm{cm})$ & $180 \pm 6$ & \\
Weight $(\mathrm{kg})$ & $79.2 \pm 13$ & \\
& & \\
\hline LP2 & P1 Group & Cr Group \\
& $(\mathrm{n}=3)$ & $(\mathrm{n}=3)$ \\
\hline & $23 \pm 5$ & $25 \pm 9$ \\
Age (yr) & $171 \pm 14$ & $174 \pm 9$ \\
Height $(\mathrm{cm})$ & $72.7 \pm 12$ & $74.1 \pm 12$ \\
Weight (kg) & & \\
& & \\
\hline LP3 & P1 Group & Cr Group \\
& $(\mathrm{n}=11)$ & $(\mathrm{n}=12)$ \\
\hline & $31 \pm 8$ & $31 \pm 6$ \\
Age (yr) & $177 \pm 6$ & $177 \pm 5$ \\
Height (cm) & $75.2 \pm 7$ & $75.0 \pm 6$ \\
Weight (kg) & & \\
& &
\end{tabular}

Table 1. The physical characteristics of subjects in each loading protocol. Data presented as the mean \pm s.d.

had similar taste, texture and appearance and were placed in generic water bottles to ensure double blind administration. On each of the test days subjects ingested a further $500 \mathrm{ml}$ of water 1 hour prior to the test in an attempt to ensure subjects were euhydrated [26].

\subsubsection{Loading protocol 2 (LP2)}

Subjects $(n=6)$ were matched for body mass then randomised in a double blind fashion to either a $\mathrm{Cr}$ or a placebo group. Subjects in both groups were assessed pre- and post-testing during two separate supplementation regimens, with one week free from any supplementation between each regimen (i.e. a total of 4 tests). There was no crossover between subject groups. Each supplementation period started on the day after the presupplementation test and finished on the day of the post-supplementation test. $\mathrm{Cr}$ supplementation consisted of $11.4 \mathrm{~g}$ of $\mathrm{Cr} \cdot \mathrm{H}_{2} \mathrm{O}$ (equivalent to $10 \mathrm{~g} \mathrm{Cr}$ ) and $70 \mathrm{~g}$ of glucose polymer made up in $1 \mathrm{~L}$ of warm water and consumed twice daily for 6 days and once more 3 hours prior to testing. The placebo supplement consisted of $85 \mathrm{~g}$ of glucose polymer in $1 \mathrm{~L}$ of warm water and consumed twice daily for 6 days and once more 3 hours prior to testing. During the first supplementation regimen, subjects in both groups also received either $1 \mathrm{~g}$ Gly $\cdot \mathrm{kg}^{-1}$ body mass or an equivalent amount of placebo (i.e. sucrose) diluted in each $1 \mathrm{~L}$ supplement before receiving the opposite during the second supplementation regimen. Therefore, four possible combinations of supplements were administered: Placebo group - placebo/placebo and placebo/Gly; Cr Group - Cr/placebo and $\mathrm{Cr} / \mathrm{Gly}$. On each of the four test days, subjects ingested a further $500 \mathrm{ml}$ of water 1 hour prior to testing.

\subsubsection{Loading protocol 3 (LP3)}

Subjects $(n=23)$ completed LP3 which was identical to the LP2 protocol outlined above with the exception that subjects began consuming their final supplement 5 hours prior to each test with instructions to complete ingestion within one hour.

\subsection{Procedures}

Subjects reported to the laboratory on the day of testing following a 3 hour fast 
and having refrained from alcohol, caffeine and strenuous exercise the day before. Upon arrival at the laboratory, height and nude body mass were measured and body water compartments estimated using a Bodystat Multiscan 5000 Bioimpedance analyser (Bodystat Ltd, Isle of Man). This method allows TBW and ECW to be estimated, and ICW to be deduced. The bioimpedance measurements were taken after the subjects had lain comfortably in a supine position for $10 \mathrm{~min}$ on a non-conductive surface with their arms and legs slightly abducted. Estimation of TBW by bioimpedance is reliable and valid when subjects are euhydrated [27]. Several studies have successfully utilised this technique in order to estimate hyperhydration induced changes in TBW $[18,23]$. Furthermore, the change in body mass from pre- to post-supplementation was determined to provide a further indirect measurement of the volume of fluid retained. Blood was obtained in all subjects from a superficial vein on the dorsal surface of the hand after standardising for postural changes (i.e. seated position for $15 \mathrm{~min}$ ).

\subsection{Blood treatment and analysis}

Blood was drawn into dry syringes and 6 $\mathrm{ml}$ dispensed into a tube containing $\mathrm{K}_{3}$ EDTA and the remaining $4 \mathrm{ml}$ dispensed into plain tubes. Duplicate aliquots $(400 \mu \mathrm{l})$ of whole blood from the $\mathrm{K}_{3}$ EDTA tube was rapidly deproteinised in $800 \mu \mathrm{L}$ of ice cold 0.3 $\mathrm{mol} \cdot \mathrm{l}^{-1}$ perchloric acid, centrifuged and the supernatant used for the measurement of glucose using standard enzymatic methods with spectrophotometric detection (Mira Plus, ABX Diagnostics, Montpellier, France). A further aliquot of blood was centrifuged and the plasma obtained was separated and used for the measurement of Gly [28]. The blood in tubes without anticoagulant was allowed to coagulate and then centrifuged; the serum collected was used for the measurement of osmolality by freezing point depression (Micro-osmometer 3300, Vitech Scientific, West Sussex, UK) (LP2 and LP3 only). The blood from the $\mathrm{K}_{3}$ EDTA tubes was also analysed for haemoglobin

(cyanmethaemoglobin method, Sigma, Chemical Company Ltd., Dorset, UK) and haematocrit (Hct) (conventional microhaematocrit method). All blood analyses were carried out in duplicate with the exception of Hct, which was carried out in triplicate. Plasma volume changes were calculated from changes in $\mathrm{Hb}$ and Hct relative to initial presupplementation values, assuming no change in red cell mass during the 7 day supplementation regimen [29].

\subsection{Data analysis}

Data were expressed as the mean \pm s.d. following a test for the normality of distribution. Statistical analysis was carried out using three factor mixed model ANOVA with repeated measures, followed by a simple main effects analysis for significant three way interactions (i.e. pre- vs. postsupplementation for each subject group and treatment) and simple main effects analysis for two way interactions. In addition, the magnitudes of change $(\Delta)$ in the four supplement regimens (placebo/placebo, placebo/Gly, $\mathrm{Cr} /$ placebo and $\mathrm{Cr} / \mathrm{Gly}$ ) were examined using either a two-sample or a paired $t$ test when significance was identified using the simple main effects analysis. Statistical significance was declared at $\mathrm{P}<0.05$. 


\section{RESULTS}

\subsection{Body mass and water compartments}

\subsubsection{LP1}

There was no significant change in the body mass of subjects following placebo supplementation $(\mathrm{P}=0.43)$ but increased significantly following $\mathrm{Cr} / \mathrm{Gly}$ supplementation $(1.59 \pm 0.21 \quad \mathrm{~kg})$ $(\mathrm{P}<0.01) \quad$ (Fig. 1). There were no significant changes in TBW, ECW or ICW following either placebo or $\mathrm{Cr} / \mathrm{Gly}$ supplementation compared to presupplementation values (Fig. 1).

\subsubsection{LP2}

The physical characteristics of the placebo and $\mathrm{Cr}$ groups were similar before supplementation (Table 1). In the placebo group, body mass increased significantly following Gly supplementation, with no change following placebo ( $\Delta$ body mass was greater following Gly supplementation; Fig. 1). In the $\mathrm{Cr}$ group, body mass increased significantly during both the placebo and Gly regimens (Fig. 1) with no difference in $\Delta$ body mass between regimens. TBW, ICW and ECW did not differ significantly pre- to postsupplementation in either the placebo or $\mathrm{Cr}$ groups in either supplementation regimen (Fig. 1).

\subsubsection{LP3}

The physical characteristics of the placebo and $\mathrm{Cr}$ groups were similar before supplementation (Table 1). In the placebo group, body mass increased significantly following Gly supplementation, with no change after placebo ( $\Delta$ body mass was greater following Gly supplementation; Fig. 1). In the $\mathrm{Cr}$ group, body mass increased significantly during both the placebo and
Gly regimens (Fig. 1). The combination of $\mathrm{Cr}$ and Gly lead to a significantly greater increase in body mass than when either was used alone ( $\mathrm{P}=0.02)$ (Fig. 1). There was no difference presupplementation in TBW, ICW and ECW between groups. In the placebo group, TBW and ECW increased significantly following Gly supplementation, whereas TBW and ECW were unaltered after the placebo regimen (Fig. 1). There was a significant increase in ICW in the placebo group following Gly supplementation $(\mathrm{P}=0.01)$ but not during the placebo regimen $(\mathrm{P}=0.10)$. In the $\mathrm{Cr}$ group, TBW, ICW and $\mathrm{ECW}$ increased significantly during both supplementation regimens (Fig. 1). Additionally, the increase in TBW and $\mathrm{ECW}$ in the Cr group was significantly greater following Gly supplementation than following placebo $(\mathrm{P}=0.02$ and $\mathrm{P}<0.01$, respectively) (Fig. 1).

3.2 Blood metabolite concentrations, osmolality and plasma volume changes

\subsubsection{LP1}

Plasma [glucose] was not different between pre- and post-supplementation measurements following either placebo or $\mathrm{Cr} / \mathrm{Gly}$ supplementation (Table 2). Plasma [Gly] increased significantly following $\mathrm{Cr} / \mathrm{Gly}$ supplementation compared to pre-supplementation with no change following placebo supplementation (Table 2). [Hb] and [Hct] remained unchanged from pre- to post-placebo supplementation. There was an increase in [Hb] and [Hct] following $\mathrm{Cr} / \mathrm{Gly}$ supplementation that did not reach statistical significance $(\mathrm{P}=0.13$ and $\mathrm{P}=0.08$, respectively) (Table 2). Plasma volume was not significantly altered by either supplementation regimen, although there 
was a tendency $(\mathrm{P}=0.07)$ for plasma volume to be reduced after $\mathrm{Cr} / \mathrm{Gly}$ supplementation (Fig. 2).

\subsubsection{LP2}

Plasma [glucose] was not different between pre- and post-supplementation measurements following either placebo or Gly supplementation in both the placebo and the $\mathrm{Cr}$ groups (Table 2). Plasma [Gly] increased significantly following placebo/Gly and Cr/Gly supplementation compared to presupplementation with no change following placebo/placebo or $\mathrm{Cr} /$ placebo supplementation (Table 2). Serum osmolality was also significantly higher post Gly supplementation compared to pre-supplementation in both the placebo $(\mathrm{P}<0.01)$ and $\mathrm{Cr}(\mathrm{P}<0.01)$ groups (Table $2)$. $[\mathrm{Hb}]$ and $[\mathrm{Hct}]$ remained unchanged from pre- to post-placebo/placebo and $\mathrm{Cr} /$ placebo supplementation. The small increases in $[\mathrm{Hb}]$ and [Hct] following placebo/Gly $(\mathrm{P}=0.21$ and $\mathrm{P}=0.17)$ and $\mathrm{Cr} /$ Gly $\quad(\mathrm{P}=0.18$ and $\mathrm{P}=0.11)$ supplementation did not reach statistical significance (Table 2). Plasma volume was not significantly altered following either placebo/placebo or $\mathrm{Cr} /$ placebo supplementation (Fig. 2), whereas both placebo/Gly and $\mathrm{Cr} / \mathrm{Gly}$ supplementation resulted in a small (2-3\%) nonsignificant reduction in plasma volume ( $\mathrm{P}=0.11$ and $\mathrm{P}=0.09$ respectively) (Fig. 2).

\subsubsection{LP3}

Plasma [glucose] was not different between groups or following Gly supplementation (Table 2). Plasma [Gly] was significantly higher post Gly supplementation compared to presupplementation in both the placebo $(\mathrm{P}<0.01)$ and $\mathrm{Cr}(\mathrm{P}<0.01)$ groups (Table $2)$. Additionally, serum osmolality was significantly higher post Gly supplementation compared to presupplementation in both the placebo $(\mathrm{P}=0.02)$ and $\mathrm{Cr}(\mathrm{P}<0.01)$ groups (Table 2). $[\mathrm{Hb}]$ and $[\mathrm{Hct}]$ remained unchanged from pre-to post-supplementation after both placebo/placebo and $\mathrm{Cr} /$ placebo supplementation (Table 2). The increase in $[\mathrm{Hb}]$ and $[\mathrm{Hct}]$ was small and nonsignificant following placebo/Gly supplementation $(\mathrm{P}=0.18$ and $\mathrm{P}=0.06$ respectively) but statistically significant following $\mathrm{Cr} / \mathrm{Gly}$ supplementation ( $\mathrm{P}=0.01$ and $\mathrm{P}=0.04$ respectively) (Table 2). Plasma volume was not significantly altered following either placebo/placebo or $\mathrm{Cr} /$ placebo supplementation (Fig. 2). Both placebo/Gly and Cr/Gly supplementation resulted in an increase in plasma volume, although only after the $\mathrm{Cr} /$ Gly supplementation did this increase reach statistical significance $(\mathrm{P}=0.06$ and $\mathrm{P}=0.01$, respectively) (Fig. 2).

\subsection{Side effects}

In general, subjects tolerated the supplementation protocol well with only one report of gastrointestinal distress after Gly supplementation during LP3.

\section{DISCUSSION}

Supplementation with combined $\mathrm{Cr}$ and Gly using the LP3 hyperhydration regimen significantly increased body mass, TBW and plasma volume (Figs. 1 and 2). Despite no significant increase in TBW and plasma volume after $\mathrm{Cr} / \mathrm{Gly}$ ingestion during LP1 and LP2 (Figs. 1 and 2), there was an increase in body mass, suggesting a significant retention of fluid. This may be explained by water requiring greater than 3 hours from ingestion of the final $\mathrm{Cr}$ /Gly supplement to disperse throughout the body water 
LP1

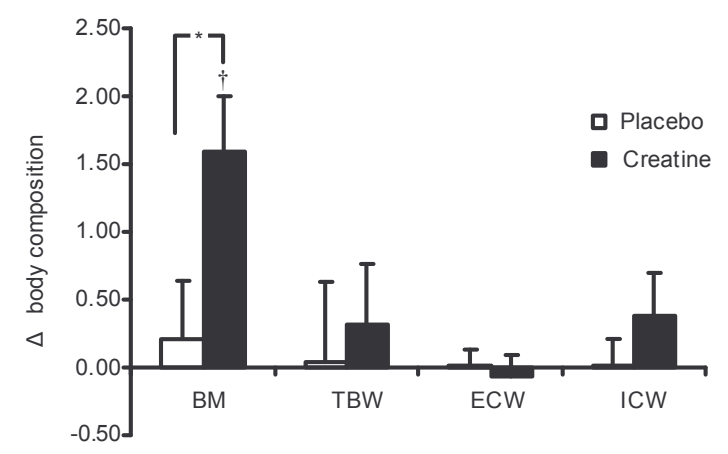

LP2
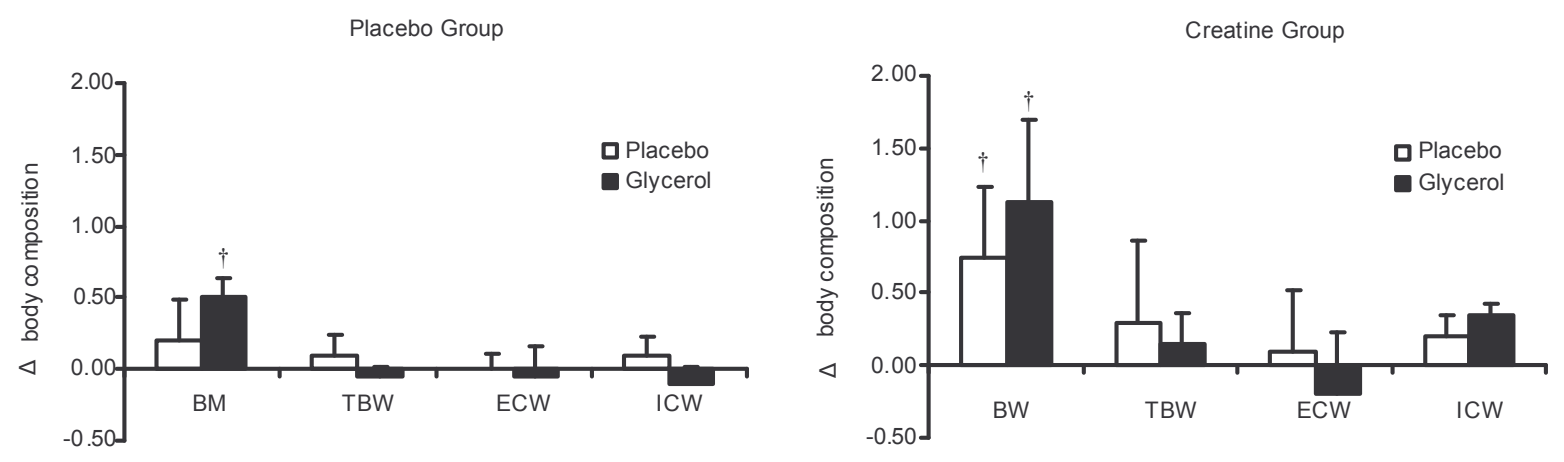

LP3
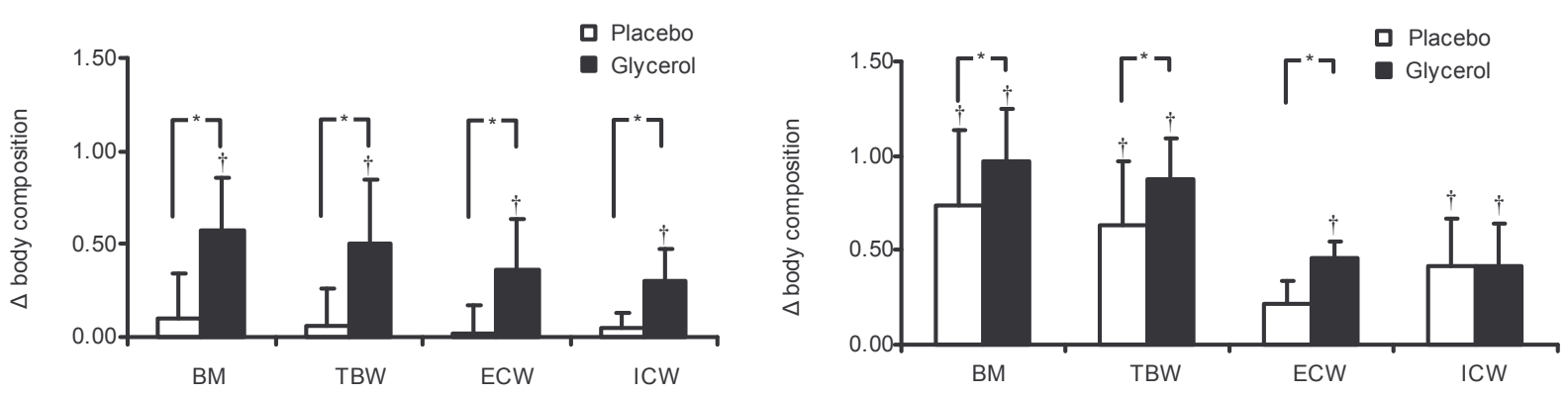

Figure 1. Changes in body mass (BM), total body water (TBW), intra-cellular water (ICW) and extra-cellular water (ECW) in the two groups. Data presented as the mean \pm s.d. $\dagger$ : indicates a significant difference pre- vs. post-supplementation. *: indicates a significant greater change $(\Delta)$ in the Gly supplementation regimen compared to the placebo supplementation regimen.

compartments. Subjects experienced a body mass increase of $1.59 \pm 0.21 \mathrm{~kg}$ after 7 days of supplementation with $\mathrm{Cr} /$ Gly in LP1 and $1.20 \pm 0.37 \mathrm{~kg}$ in LP2 (Fig. 1). This exceeds the $0.4-1.0 \mathrm{~kg}$ increase in body mass observed in other hyperhydration studies [17,18,21]. This increase in body mass was not accompanied by an increase in TBW indicating an increase in water retention that was not measured by bioimpedance analysis. These results are not in 
agreement with other hyperhydration studies that demonstrated an increase in fluid retention as reflected by a significant increase in TBW $[18,19,23]$. For example, subjects in the study by Kilduff et al. [18] experienced a $0.8 \mathrm{~kg}$ increase in body mass accompanied by a $0.6 \mathrm{~L}$ increase in TBW after 7 days of $\mathrm{Cr}$ supplementation. Conversely, combined Cr/Gly supplementation following LP3 resulted in a significant $0.97 \pm 0.28 \mathrm{~kg}$ increase in body mass accompanied by a $0.87 \pm 0.21 \mathrm{~L}$ increase in TBW. The results from LP3 also demonstrate that both the increase in body mass and the volume of water retained by ingesting either $\mathrm{Cr}$ or Gly can be significantly enhanced by combining these two hyperhydrating agents. The $870 \mathrm{ml}$ (range 600-1400 $\mathrm{ml}$ ) increase in TBW resulting from ingestion of this novel "water-loading" strategy that combines $\mathrm{Cr}$ and Gly, was significantly larger than the volume retained with either $\mathrm{Cr}$ or Gly alone (Fig. 1). Additionally, the retained water was dispersed equally between the intra- and extra-cellular water compartments (Fig. 1). It seems plausible that the water retained by combining $\mathrm{Cr}$ and Gly was mediated via a Cr-induced increase in ICW and a Glyinduced increase in ECW. This innovative "water-loading" strategy comprised of two agents that specifically target both ICW and ECW compartments and as such, may overcome the limitations of previous hyperhydration strategies. For example, Gly has been investigated as a potential hyperhydrating agent for a number of decades [17,22]. However, the benefits of Gly hyperhydration are equivocal with at least 23 original papers published on Gly hyperhydration providing conflicting results. Where
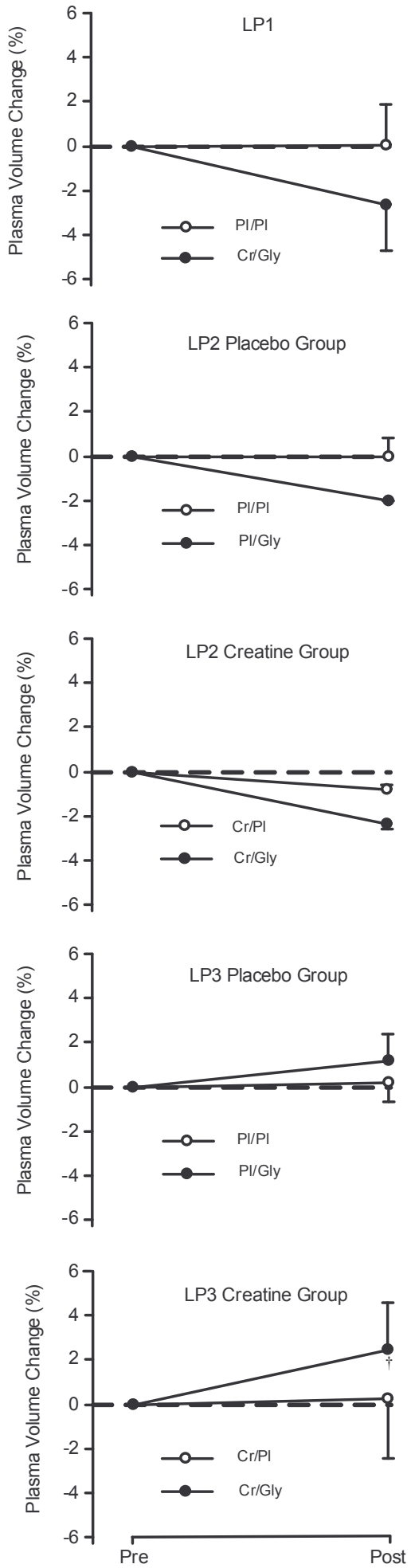

Figure 2. Changes in plasma volume between pre- and post supplementation in each subject group and loading protocol. Data presented as the mean \pm s.d. $\dagger$ : indicates a significant difference pre- vs. post-supplementation. 


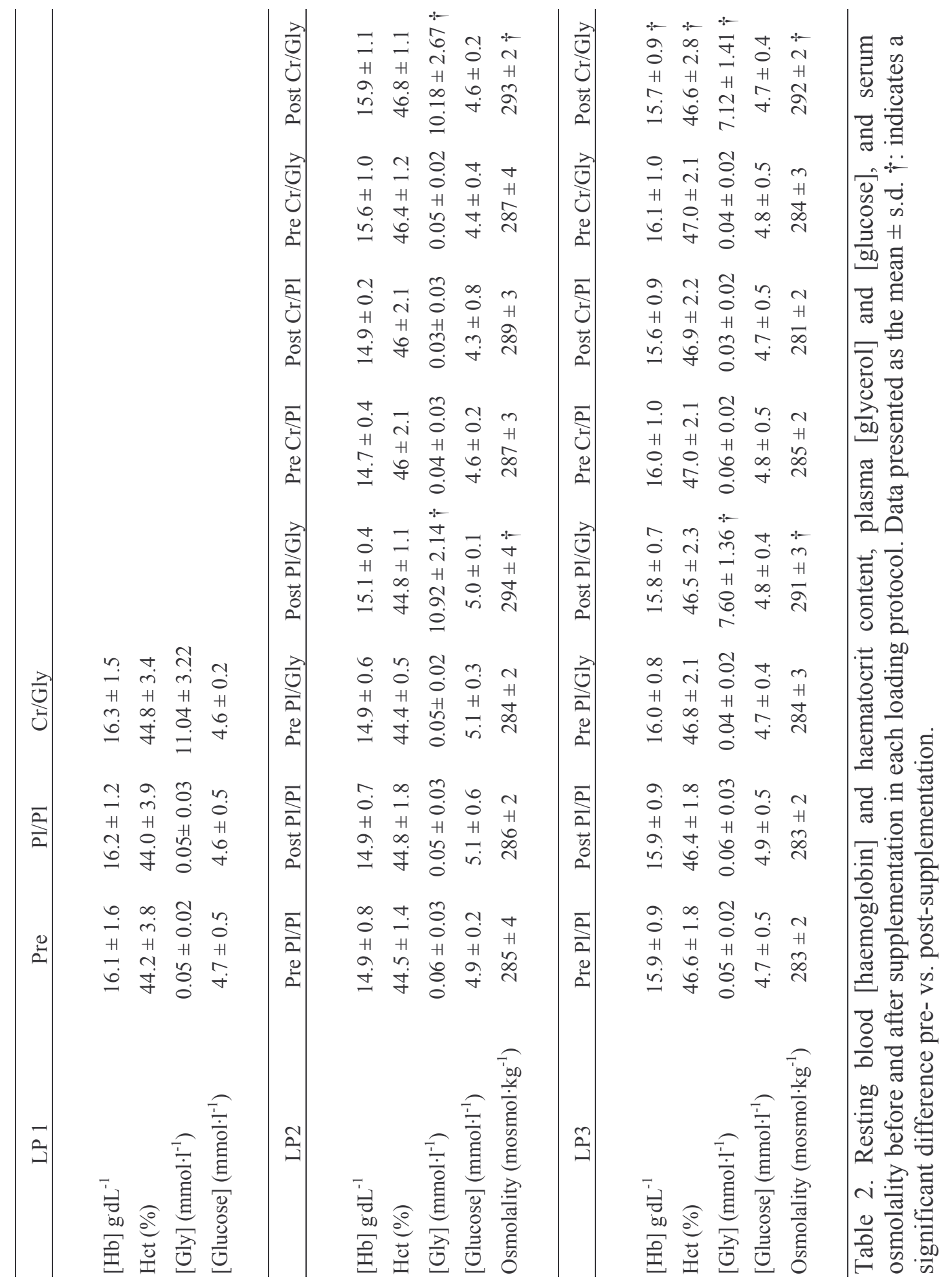


hyperhydration was induced, the hydrating effects were transient due to the metabolism of Gly by the liver and kidneys. Of greater concern is the potential side-effect of cerebral dehydration, due to the relatively impermeability of the blood-brain barrier to Gly, with resulting headaches [30]. Combining Gly with $\mathrm{Cr}$ may overcome this, as in contrast to Gly, $\mathrm{Cr}$ is taken up by the brain [31] and could counteract the effects of Gly.

A key finding of the present series of studies is the importance of methodology that led to the differences of changes in TBW between LP2 and LP3. The only difference between LP2 and LP3 was the length of time between the ingestion of the final supplement and testing. Thus, it would seem apparent that a period of time in excess of 3 hours is required for the retained water to be distributed within body water compartments. Previous studies examining the effectiveness of Gly supplementation as a hyperhydration method have consistently utilised a single Gly bolus mixed with water and ingested between 2-3 hours prior to analysis [17,21]. However, when Gly was delivered in a similar fashion alongside a $\mathrm{Cr}$ hyperhydration protocol previously shown to be successful in our lab [18], no increase in hydration was measured (Fig. 1). Previous Gly hyperhydration studies have quantified water retention by the volume of urine produced [16,22], which provides no information as to where the retained water was distributed. Body compartment analysis by multifrequency bioimpedance combined with changes in body mass used in the present study, provides data indicating fluid changes in both the intra- and extra-cellular water compartments. Furthermore, the mechanism by which bioimpedance estimates body water provides insight into the confounding data from LP1 and LP2. Since hypertonic solutions such as the $\mathrm{Cr} /$ Gly combination $(965 \pm 61$ mosmol $\cdot \mathrm{kg}^{-1}$ ) cause an initial net movement of fluid into the intestinal lumen [32], there is a loss of ECW and thus TBW, which ultimately leads to dehydration, albeit temporarily. This is confirmed by the small reductions in plasma volume that occurred after supplementation with both $\mathrm{Pl} / \mathrm{Gly}$ and Cr/Gly in LP1 and LP2. Interestingly, fluid changes in the trunk have little effect on bioimpedance measurements as the trunk only accounts for $5-12 \%$ of total body impedance [33]. This is confirmed by the relatively small impact on bioimpedance measurements of up to $2 \mathrm{~L}$ of fluid within the abdominal cavity [34]. Additionally, the profoundly high osmolality of the $\mathrm{Cr} / \mathrm{Gly}$ solution may have inhibited gastric emptying [35], further contributing to the lack of increase in TBW 2-3 hours after Cr/Gly ingestion as demonstrated in LP1 and LP2. Although $\mathrm{Cr} / \mathrm{Gly}$ supplementation results in significant water retention, a period of time greater than 3 hours is required after ingestion of the final $\mathrm{Cr} / \mathrm{Gly}$ supplement before significant hydrating effects are discerned throughout the body water compartments. Furthermore, it is possible that a similar effect would have been observed after LP1 and LP2 had a longer period of time been left between ingestion of the final supplement and testing. This is in contrast to the majority of previous studies that utilised $\mathrm{Cr}$ or Gly alone to hyperhydrate, which concluded that there were no changes in plasma volume compared to water or a placebo [e.g. 1618]. Similarly to these previous studies, there was no appreciable change in plasma volume in the present study in LP3 from pre- to post-supplementation 
when either $\mathrm{Cr}$ or Gly alone were ingested (Fig. 2). However, the fact that combined $\mathrm{Cr}$ and Gly resulted in a significantly greater retention of fluid than $\mathrm{Cr}$ or Gly alone and the unique way in which this novel hyperhydration strategy retains fluid, may account for these notable differences. Plasma volume can decrease during space travel by as much as $17 \% \quad[2,3,8]$. The $2.4 \%$ expansion induced by $\mathrm{Cr}$ and Gly in the present study may not be large enough to offset this deficit. Further research is required to examine whether plasma volume expansion via $\mathrm{Cr}$ and Gly hyperhydration can minimise the adverse effects of plasma volume reduction during space travel and orthostatic intolerance on return to earth.

Despite the relatively minor increases in plasma volume associated with combined creatine and glycerol ingestion, astronauts encounter several other physiological problems during space travel that this novel hyperhydration strategy may potentially counteract. For example, the effect of plasma volume loss in space may result in excessive heat strain for astronauts wearing protective garments during launch and landing. Dehydrated ( $>$ $3 \%$ body mass) unacclimatised individuals will exhibit excessive heat strain (core temperature exceeding $39^{\circ} \mathrm{C}$ ) during the pre-launch and launch of the space shuttle [36]. Core temperature has been measured in crewmembers during landing and found to be significantly elevated to approximately $38^{\circ} \mathrm{C}$ despite the use of a liquid cooling garment [37]. Recent studies completed in this lab suggest that ingestion of $\mathrm{Cr}$ and Gly can significantly reduce cardiovascular and thermal strain during exercise induced heat stress [38]. Potentially this could improve physical and mental performance during space travel and offer astronauts some protection from heat injury. Additionally, $\mathrm{Cr}$ supplementation prior to and during prolonged periods of immobilisation (hypokinesia) significantly attenuated skeletal muscle atrophy [39]. Hypokinesia encountered during microgravity has been shown to induce muscle atrophy, slow-to-fast twitch muscle fibre shift and a decrease in force generation capacity $[39,40]$. One of the proposed mechanisms for the protective effect of $\mathrm{Cr}$ on skeletal muscle includes higher mitotic activity in satellite cells [41]. In addition, it is also possible that $\mathrm{Cr}$ is able to activate signalling pathways, protecting skeletal muscle against proteolysis [42]. Alternatively, the increase in cellular hydration that occurs after $\mathrm{Cr}$ supplementation (Fig. 1) may also have a role in controlling protein turnover as this can act as a signal, stimulating protein synthesis and attenuating protein breakdown [43]. However, further research is required to examine the exact effects of $\mathrm{Cr}$ on skeletal muscular atrophy alongside a structured weight-training program during prolonged space travel.

\section{CONCLUSIONS}

This study has demonstrated that supplementation with combined $\mathrm{Cr}$ and Gly significantly increased body mass, TBW and plasma volume. The timing of ingestion is critical, with the final supplement requiring to be consumed approximately 5 hours prior to the need for hyperhydration (e.g. prior to take-off and landing). Although $\mathrm{Cr} / \mathrm{Gly}$ appear to have a synergistic effect on hydration status and thermoregulation in a terrestrial environment, it remains to be determined whether this translates into 
minimising the adverse physiological effects of microgravity.

\section{ACKNOWLEDGEMENTS}

This study was supported by a grant from Iovate Health Sciences Research Inc. The authors acknowledge John Wilson, Heather Collin and Annie Keenan for their excellent technical assistance. The cooperation of the subjects is greatly appreciated.

\section{REFERENCES}

1. Lane, H. W. and Feeback, D. L., Water and energy dietary requirements and endocrinology of human space flight. Nutrition, 2002, 18, 820-828.

2. Leach, C. S., Alfrey, C. P., Suki, W. N., Leonard, J. I., Rambaut, P. C., Inners, L. D., Smith, S. M., Lane, H. W. and Krauhs J. M., Regulation of body fluid compartments during short-term spaceflight. Journal of Applied Physiology, 1996, 81, 105116.

3. Smith, S. M., Krauhs, J. M. and Leach, C. S., Regulation of body fluid volume and electrolyte concentrations in spaceflight. Advances in Space Biology and Medicine, 1997, 6, 12365.

4. Grigoriev, A. I., Huntoon, C. L., Morukov, B. V., Lane, H. W., Larina, I. M. and Smith, S. M., Endocrine, renal, and circulatory influences on fluid and electrolyte homeostasis during weightlessness: a joint Russian-U.S. project. Journal of Gravitational Physiology, 1996, 3, 83 86.

5. Leach, C. S., Alexander, W. C. and Johnson, P. C., Endocrine, electrolyte, and fluid volume changes associated with Apollo missions. In Biomedical results of Apollo, eds. R. S. Johnston, L. F. Dietlein and C. A. Berry. National Aeronautics and Space Administration, Washington DC, 1975, pp. 163.

6. Leach, C. S. and Rambaut, P. C., Biochemical responses of the Skylab crewmembers. In Biomedical results from Skylab, eds. R. S. Johnston and L. F. Dietlein. National Aeronautics and Space Administration, Washington DC, 1977, pp. 204.

7. Thornton, W. E., Hoffler, G. W. and Rummel, J. A., Anthropometric changes and fluid shifts. In Biomedical results from Skylab, eds. R. S. Johnston and L. F. Dietlein. National Aeronautics and Space Administration, Washington DC, 1977, pp. 330-338.

8. Lane, H. W., Leach, C. S. and Smith S. M., Fluid and electrolyte homeostasis. In Nutrition in spaceflight and weightlessness models, eds. H. W. Lane and D. A. Schoeller. CRC Press, Florida, 2000, pp. 119.

9. Seddon, M. R., Fettman, M. J. and Phillips, R. W., Practical and clinical nutritional concerns during space flight. American Journal of Nutrition, 1994, 60, 825S-830S.

10. Fortney, S. M., Mikhaylov, V., Lee, S. M., Kobzev, Y., Gonzalez, R. R. and Greenleaf, J. E. Body temperature and thermoregulation during submaximal exercise after 115-day spaceflight. Aviation, Space and Environmental Medicine, 1998, 69, 137-41.

11. Buckey, J. C. Jr., Lane, L. D., Levine, B. D., Watenpaugh, D. E., Wright, S. J., Moore, W. E., Gaffney, F. A. and Blomqvist, C. G., Orthostatic 
intolerance after spaceflight. Journal of Applied Physiology, 1996 ${ }^{\mathrm{a}}, \mathbf{8 1}, 7-$ 18.

12. Buckey, J. C. Jr., Gaffney, F. A., Lane, L. D., Levine, B. D., Watenpaugh, D. E., Wright, S. J., Yancy, C. W. Jr., Meyer, D. M. and Blomqvist, C. G., Central venous pressure in space. Journal of Applied Physiology, 1996 ${ }^{\mathrm{b}}, \mathbf{8 1}, 19-25$.

13. Convertino, V. A., Doerr, D. F. and Stein, S. L., Changes in size and compliance of the calf after 30 days of simulated microgravity. Journal of Applied Physiology, 1989, 66, 15091512.

14. Fortney, S. M., Vroman, N. B., Beckett, W. S., Permutt, S. and LaFrance, N. D. Effect of exercise hemoconcentration and hyperosmolality on exercise responses. Journal of Applied Physiology, 1988, 65, 519-524.

15. Lane, H. W. and Smith, S. M. Nutrition in space. In: Modern Nutrition in Health and Disease, eds. M. Shils, M. Shike and J. Olson, and Ross AC. Lippincott Williams \& Wilkins, New York, 1999, pp. 783788.

16. Freund, B. J., Montain, S. J., Young, A. J., Sawka, M. N., DeLuca, J. P., Pandolf, K. B. and Valeri, C. R., Glycerol hyperhydration: hormonal, renal, and vascular fluid responses. Journal of Applied Physiology, 1995, 79, 2069-2077.

17. Lyons, T. P., Riedesel, M. L., Meuli, L. E. and Chick, T. W., Effects of glycerol induced hyperhydration prior to exercise in the heat on sweating and core temperature. Medicine and Science in Sports and Exercise, 1990, 22, 77-83.

18. Kilduff, L. P., Georgiades, E., James, N., Minnion, R. H., Mitchell, M.,
Kingsmore, D., Hadjicharlambous, M. and Pitsiladis, Y. P., The effects of creatine supplementation on cardiovascular, metabolic, and thermoregulatory responses during exercise in the heat in endurancetrained humans. International Journal of Sport Nutrtion and Exercise Metabolism, 2004, 14, 443-60.

19. Seifert, J. G., Luetkemeier, W. S. and Coles, M., The effects of glycerol ingestion on fluid compartment volumes, physiological function, and cycling performance. Medicine and Science in Sports and Exercise (Supplement), 1995, 27, S19.

20. Murray, R., Eddy, D. E., Paul, G. L., Seifert, J. G. and Halaby, G. A., Physiological responses to glycerol ingestion during exercise. Journal of Applied Physiology, 1991, 71, 144149.

21. Montner, P., Stark, D. M., Riedesel, M. C., Murata, G., Robergs, R., Timms, M. and Chick, T. W., Preexercise glycerol hydration improves cycling endurance time. International Journal of Sports Medicine, 1996, 17, 27-33.

22. Anderson, M. J., Cotter, J. D., Garnham, A. P., Casley, D. J. and Febbraio, M. A., Effect of glycerolinduced hyperhydration on thermoregulation and metabolism during exercise in the heat. International Journal of Sport Nutrition and Exercise Metabolism, 2001, 11, 315-333.

23. Kern, M., Podewils, L. J., Vukovich, M. and Buono, M. J., Physiological response to exercise in the heat following creatine supplementation., Journal of Exercise Physiology Online, 2001, 4, 18-27.

24. Vandenberghe, K., Gillis, N., Van Leemputte, M., Van Hecke, P., 
Vanstapel, F., and Hespel, P. Caffeine counteracts the ergogenic action of muscle creatine loading. Journal of Applied Physiology, 1996, 80, 452457.

25. Harris, R. C., Sodehind, K., and Hultman, E. Elevation of Creatine in resting and exercised muscle of normal subjects by $\mathrm{Cr}$ supplementation. Clinical Science, 1992, 83, 367-374.

26. Convertino, V.A., L.E. Armstrong, E.F. Coyle, G.W. Mack, M.N. Sawka, L.C. Senay Jr, and W.M. Sherman. American College of Sports Medicine Position Stand: Exercise and fluid replacement. Medicine and Science in Sports and Exercise, 1996, 28, i-vii.

27. O'Brien, C., Young, A. J., and Sawka, M. N. Bioelectrical impedance to estimate changes in hydration status. International Journal of Sports Medicine, 2002, 23, 361-366.

28. Boobis, L. H., and Maughan, R. J. A simple one-step enzymatic fluorometric method for the determination of glycerol in 20 microliters of plasma. Clinica Chimica Acta, 1983, 132, 173-179.

29. Dill, D. B., and Costill, D. L. Calculations of percentage changes in volumes of blood, plasma, and red cells in dehydration. Journal of Applied Physiology, 1974, 37, 247248.

30. Tourtellotte, W.W., J.L. Reinglass, and T.A. Newkirk. Cerebral dehydration action of glycerol. Clinical Pharmacology and Therapeutics, 1972, 13, 159-171.

31. Matthews, R. T., Ferrante, R. J., Klivenyi, P., Yang, L., Klein, A. M., Mueller, G., Kaddurah-Daouk, R., and Beal, M. F. Creatine and cyclocreatine attenuate MPTP neurotoxicity.
Experimental Neurology, 1999, 157, 142-149.

32. Gisolfi, C.V., R.W. Summers, H.P. Schedl, T.L. Bleiler, and R.A. Oppliger. Human intestinal water absorption: direct vs. indirect measurements. American Journal of Physiology, 1990, 258, G216-G222.

33. Kushner, R. F. Bioelectrical impedance analysis: a review of principles and applications. Journal of the American College of Nutrition, 1992, 11, 199-209.

34. Kushner R.F., Gudivaka R., Schoeller D.A. Clinical characteristics influencing bioelectrical impedance analysis measurements. American Journal of Clinical Nutrition, 1996, 64, 423S-427S.

35. Costill, D. L., and Saltin, B. Factors limiting gastric emptying during rest and exercise. Journal of Applied Physiology, 1974, 37, 679-683.

36. Pandolf, K. B., Stroschlein, L. A., Gonzalez, R. R., and Sawka, M. N. Predicting human heat strain and performance with applications to space operations. Aviation, Space and Environmental Medicine, 1995, 66, 364-368.

37. Rimmer, D. W., Djik, D. J., Ronda, J. M., Hoyt, R., and Pawelczyk, J. A. Efficacy of liquid cooling garments to minimize heat strain during Space Shuttle deorbit and landing. Medicine and Science in Sports and Exercise, 1999, 31, S305.

38. Easton, C., Turner, S. and Pitsiladis, Y. P. Creatine and glycerol hyperhydration in trained subjects prior to exercise in the heat. International Journal of Sports Nutrition and Exercise Metabolism. 2006, In press.

39. Aoki, M. S., Lima, W. P., Miyabara, E. H., Gouveia, C. H. and Moriscot, 
A. S., Deleteriuos effects of immobilization upon rat skeletal muscle: role of creatine supplementation. Clinical Nutrition, 2004, 23, 1176-1183.

40. Caiozzo, V. J., Baker, M. J., Herrick, R. E., Tao, M., and Baldwin, K. M. Effect of space flight on skeletal muscle: mechanical properties and MHC content of a slow muscle. Journal of Applied Physiology, 1994, 76, 1764-1773.

41. Dangott, B., Schultz, E., and Mozdziak, P. E. Dietary creatine monohydrate supplementation increases satellite cell mitotic activity during compensatory hypertrophy. International Journal of Sports Medicine, 1999, 20, 13-26.

42. Vierck, J. L., Icenoggle, D. L., Bucci, L., and Dodson, M. V. The effects of ergogenic compounds on myogenic satellite cells. Medicine and Science in Sports and Exercise, 2003, 35, 769776.

43. Haussinger, D., Roth, E., Lang, F., and Gerok, W. Cellular hydration state: an important determinant of protein catabolism in health an disease. Lancet, 341 (8856), 13301332. 